\title{
Hyaluronic-Coated Albumin Nanoparticles for the Non-Invasive Delivery of Apatinib in Diabetic Retinopathy
}

\author{
Salma El-Sayed Radwan' \\ Amal El-Kamel (iD' \\ Eiman I Zaki iD ${ }^{2}$ \\ Susi Burgalassi $\mathbb{D}^{3}$ \\ Erica Zucchetti $\mathbb{1}^{3}$ \\ Riham M El-Moslemany' \\ 'Department of Pharmaceutics, Faculty of \\ Pharmacy, Alexandria University, \\ Alexandria, Egypt; ${ }^{2}$ Department of \\ Histology and Cell Biology, Faculty of \\ Medicine, Alexandria University, \\ Alexandria, Egypt; ${ }^{3}$ Department of \\ Pharmacy, University of Pisa, Pisa, Italy
}

Purpose: Apatinib (Apa) is a novel anti-vascular endothelial growth factor with the potential to treat diabetic retinopathy (DR); a serious condition leading to visual impairment and blindness. DR treatment relies on invasive techniques associated with various complications. Investigating topical routes for Apa delivery to the posterior eye segment is thus promising but also challenging due to ocular barriers. Hence, the study objective was to develop Apa-loaded bovine serum albumin nanoparticles (Apa-BSA-NPs) coated with hyaluronic acid (HA); a natural polymer possessing unique mucoadhesive and viscoelastic features with the capacity to actively target CD44 positive retinal cells, for topical administration in DR.

Methods: Apa-BSA-NPs were prepared by desolvation using glutaraldehyde for crosslinking. HA-coated BSA-NPs were also prepared and HA: NPs ratio optimized. Nanoparticles were characterized for colloidal properties, entrapment efficiency (EE\%), in vitro drug release and mucoadhesive potential. In vitro cytotoxicity on rabbit corneal epithelial cells (RCE) was assessed using MTT assay, while efficacy was evaluated in vivo in a diabetic rat model by histopathological examination of the retina by light and transmission electron microscopy. Retinal accumulation of fluorescently labeled BSA-NP and HA-BSANP was assessed using confocal microscope scanning.

Results: Apa-HA-BSA-NPs prepared under optimal conditions showed size, PdI and zeta potential: $222.2 \pm 3.56 \mathrm{~nm}, 0.221 \pm 0.02$ and $-37.3 \pm 1.8 \mathrm{mV}$, respectively. High EE\% (69 $\pm 1 \%)$, biphasic sustained release profile with an initial burst effect and mucoadhesion was attained. No evidence of cytotoxicity was observed on RCE cells. In vivo histopathological studies on DR rat model revealed alleviated retinal micro- and ultrastructural changes in the topical HAApa-BSA-NP treated eyes with normal basement membrane and retinal thickness comparable to normal control and intravitreally injected nanoparticles. Improved retinal accumulation for HA-BSA-NP was also observed by confocal microscopy.

Conclusion: Findings present HA-Apa-BSA-NPs as a platform for enhanced topical therapy of DR overcoming the devastating ocular complications of the intravitreal route.

Keywords: retinopathy, apatinib, bovine serum albumin, hyaluronic, mucopenetration, active targeting

\section{Introduction}

Diabetic retinopathy (DR) is a microvascular complication of diabetes and is the fourth leading cause of visual impairment and blindness world-wide. ${ }^{1}$ Nowadays, DR therapy depends mainly on invasive techniques such as intravitreal injections. One of the major drawbacks of intravitreal injections is patient discomfort and poor compliance worldwide. Moreover, they may result in ocular complications such as
Correspondence: Riham M El-Moslemany I, Khartoum Square, Azarita, Alexandria, Egypt

Tel + I I 201006020405

Email riham.elmoslemany@alexu.edu.eg 
intraocular bleeding and endophthalmitis. Topical ophthalmic dosage forms such as solutions, suspensions, ointments, gels, or emulsions have shown promising outcomes in treating anterior eye diseases. ${ }^{2}$ Delivering drugs to the posterior region of the eye remains a challenge up to this day. ${ }^{3}$ The unique structure of the eye renders the passage of drugs extremely difficult as it combats multiple barriers of various properties. ${ }^{4}$ Topical delivery to the posterior of the eye faces many challenges; the cornea, conjunctiva, sclera, Bruch's membrane and retinal pigment epithelium act as static barriers. ${ }^{5}$ On the other hand, tear fluid, lymphatic vessels, and conjunctival and choroidal blood flow act as a dynamic barrier. Therefore, owing to these barriers, the bioavailability of drugs administered topically to the posterior segment of the eye is $5 \%$ in the aqueous humor and less than that in the vitreous humor. ${ }^{6}$ The design of topical dosage forms that enhance the ocular bioavailability of drugs at the posterior of the eye has become very crucial.

Ocular drug delivery systems in the nano-range provide the advantage of protecting the cargo from external degradation, sustaining drug release, penetrating physiological barriers, and delivering the drug to specific cells by targeting. ${ }^{7}$

Albumin is an extensively used macromolecule because of its natural properties including biodegradability and biocompatibility. ${ }^{8,9}$ Preparation of albumin NPs is relatively easy, and takes place under mild conditions either by emulsification, desolvation, complex coacervation, and electrospray. ${ }^{9}$ Moreover, carboxyl and amine functional groups found on the surface of albumin, play a role in surface modification and, hence targeting. ${ }^{10}$ This in turn enhances cellular uptake across the endothelial cells. $^{11}$

Kim et al. ${ }^{12}$ reported that albumin formulations could provide a useful system to deliver various cytoprotectants to the eye anterior or posterior regions to prevent ocular diseases. $^{12}$ Bovine serum albumin nanoparticles (BSANPs) have been reported to have the ability to permeate the rabbit's cornea to deliver acyclovir to deep corneal layers without irritating the eye. ${ }^{13}$ Das et al. ${ }^{14}$ documented also that aspirin-loaded BSA-NPs had the potential to topically treat posterior eye diseases such as DR.

Surface coating of albumin nanoparticles with hyaluronic (HA) acid has gained a lot of interest in the field of ocular drug delivery. ${ }^{15}$ HA hygroscopic characteristic enables it to form hydrogen bonds with mucin despite its anionic nature. ${ }^{16} \mathrm{HA}$ is a biodegradable, non- immunogenic biopolymer, and might be an interesting molecule for targeted ocular delivery since it is a major constituent of the vitreous humor. It is found throughout the retina and has the ability to interact with Cluster of differentiation (CD44) receptors on the surface of many retinal cell types. ${ }^{17}$ It has been reported that HA-coated albumin NPs loaded with Cx43 mimetic peptide, exhibited enhanced in vitro cellular uptake and ex vivo retinal penetration, compared to uncoated particles, via HA-CD44 receptor mediated interactions. ${ }^{18,19}$ Moreover, degradation of endothelial HA could be observed in DR pathophysiology. Therefore, maintaining HA could prevent further diabetic vascular complications and organ damage. ${ }^{20}$

Apatinib, (Apa) a novel and selective inhibitor of VEGF receptor 2, has been demonstrated recently to exhibit anticancer efficacy by inhibiting the VEGF-signaling pathway. ${ }^{21,22} \mathrm{Kim}$ and $\mathrm{Suh}^{23}$ investigated its antiangiogenic effect on oxygen-induced retinopathy. This was explained by its selective binding to VEGF receptor 2 that plays a crucial role in angiogenesis by stimulating auto-phosphorylation at the carboxy terminal tail and kinase-insert region, which is the most pro-angiogenic effect. $^{23}$ Intravitreal injection of Apa attenuated retinal neovascularization in mice while proving to be non-toxic. Apa is insoluble in water; hence, nanoparticle encapsulation avoids accumulation of the insoluble drug in the vitreous cavity, which is often associated with ocular toxicity. $^{24}$ Lee et al. $^{22}$ documented that Apa-human serum albumin (HSA)-conjugated polyethylene glycol (PEG) nanoparticles, are promising for the prevention and treatment of corneal neovascularization-related ocular disorders. In addition, intravitreal injection of Apa-HSAPEG nanoparticles formulated by Jeong et al. inhibited retinal vascular leakage in STZ-injected diabetic mice. ${ }^{24}$

The objective of the current study is to develop topically delivered apatinib loaded bovine serum albumin nanoparticles coated with hyaluronic acid to achieve improved delivery to retinal cells for treatment of diabetic retinopathy.

\section{Materials and Methods Materials}

Apatinib was purchased from ATK chemical (QingPu, Shanghai, China). Bovine serum albumin was obtained from (Biowest, USA). Hyaluronic acid sodium salt (Mwt $1.4 * 10^{6} \mathrm{Da}$ ) was provided by Euromedex (Strasbourg, France). Glutaraldehyde was from Al-Gomhureya 
chemicals (Egypt). Hexadecyltrimethyl ammonium bromide (CTAB) and porcine mucin were purchased from Sigma Aldrich (USA). Ethanol and Acetonitrile, HPLC grade were from Fisher Scientific (UK). All other chemicals and organic solvents were of analytical grade.

\section{Preparation of BSA-NPs and HA-BSA- NPs}

BSA-NPs were prepared by the well-established desolvation method. ${ }^{25}$ Briefly, BSA $(25,50$ and $100 \mathrm{mg}$ ) was dissolved in $1 \mathrm{~mL}$ of $10 \mathrm{mM} \mathrm{NaCl}$ followed by stirring for 10 minutes. The $\mathrm{pH}$ was adjusted to 8 using 0.1 $\mathrm{N} \mathrm{NaOH}$, then, the desolvating agent, absolute ethanol, was added dropwise $(1 \mathrm{~mL} / \mathrm{min})$ till the formation of an opalescent dispersion. Subsequently, the desolvated BSANPs were cross linked with $25 \mu \mathrm{L}$ of $8 \%$ (v/v) glutaraldehyde and the reaction was kept under constant magnetic stirring for 24 hours at room temperature. The resulting colloidal dispersion was centrifuged (Sigma Laboratory Refrigerated Centrifuge, Model 3K-30; Germany) at $15,000 \mathrm{rpm}$ for 25 minutes. The supernatant was then removed, and the cross-linked precipitated BSA-NPs were redispersed in 1mL PBS ( $\mathrm{pH}$ 7.4). Apa loaded BSANPs were prepared by adding Apa (5 mg/mL in DMSO) to the BSA solution at the beginning of the process.

For the preparation of HA-coated BSA-NPs, the BSANPs dispersion $\mathrm{pH}$ was adjusted to 4.9 using $0.1 \mathrm{~N} \mathrm{HCl}$, then added dropwise to $(0.5,1$ or $2 \mathrm{~mL})$ colloidal solution of HA $(2 \mathrm{mg} / \mathrm{mL})$. The mixture was then stirred for 2 hours at $37^{\circ} \mathrm{C}$.

\section{Determination of Hyaluronic Acid Content}

The efficiency of HA coating was tested by measuring the concentration of free $\mathrm{HA}$ in the supernatant following centrifugation using the cetyltrimethyl ammonium bromide (CTAB) turbidimetric method. ${ }^{26}$ Briefly, an aliquot of $2 \mathrm{~mL}$ of CTAB reagent $(2.5 \mathrm{~g}$ CTAB in $100 \mathrm{~mL}$ of 0.2 $\mathrm{mol} / \mathrm{L} \mathrm{NaCl}$ ) was precisely added to $1 \mathrm{~mL}$ of $\mathrm{HA}$ solution and gently shaken to ensure mixing. The timer was set to accurately control reaction time. At the 10th min, sample absorbance was measured using ultraviolet spectrophotometry at $\lambda_{\max } 400 \mathrm{~nm}$. Deionized water was used as blank. HA concentration was then obtained from a calibration curve constructed using different dilutions of HA standard solution.

\section{In vitro Characterization of BSA NPs Colloidal Properties and Morphology}

The mean particle size, polydispersity index (PdI) and zeta potential of BSA-NPs were measured by Zetasizer (PCS Zetasizer $^{\circledR} \quad$ Nano ZS Series DTS 1060, Malvern Instruments S.A., Worcestershire, UK) at a fixed angle at $25^{\circ} \mathrm{C}$ using a $4 \mathrm{~mW} \mathrm{He}-\mathrm{Ne}$ laser at $633 \mathrm{~nm}$. Dispersions were diluted in deionized water and measurements were performed in triplicate.

The morphology of BSA-NPs and HA-BSA-NPs was examined by transmission electron microscopy (TEM) using JEOL, JEM-100 CX Electron Microscope, Tokyo, Japan. Before analysis, the selected dispersions were sprayed onto copper grids without staining ${ }^{27}$ and shots were taken at $\mathrm{x} 30-50 \mathrm{~K}$ magnification.

\section{Encapsulation Efficiency}

Entrapment efficiency (EE\%) was calculated by determining the concentration of free (un-entrapped) Apa in the supernatant after separation of BSA-NPs by centrifugation. A high-performance liquid chromatographic assay (HPLC-UV) was used for Apa determination as reported by Lee et al. ${ }^{22}$ Agilent Technologies-1260 infinity; Santa Clara, CA, USA was used. Separation was carried out on reversed phase $\mathrm{C} 18$ column Kinetex $^{\mathrm{TM}}$; $250 \times 4.6 \mathrm{~mm}$, particle size $5 \mu \mathrm{m}$, pore size $100 \mu \mathrm{m})$. An isocratic eluent consisting of acetonitrile and distilled water in the ratio 45:55 v/v was used. The injection volume was $20 \mu \mathrm{L}$ and the flow rate was adjusted to $0.5 \mathrm{~mL} / \mathrm{min}$. Peaks were detected at $347 \mathrm{~nm}$ using a UV detector. All solutions were protected from light and used within $24 \mathrm{~h}$. Reversedphase elution was carried out at room temperature. Apa concentration was calculated using calibration standards. Linearity was checked in the concentration range 50-5000 $\mu \mathrm{g} / \mathrm{mL}$ with a determination coefficient 0.999 . The method was validated for limit of detection (LOD) and limit of quantitation (LOQ) which were 5.77 and $19.25 \mu \mathrm{g} / \mathrm{mL}$, respectively. Measurements were done in triplicate. The \% EE of Apa in BSA-NPs was calculated from the difference between the initial drug concentration added and the free drug concentration in the supernatant using the following equation:

$$
\% E E=\frac{\left(C_{i}-C_{f}\right) \times 100}{C_{i}}
$$

where $\mathrm{C}_{\mathrm{i}}$ is the initial drug content and $\mathrm{C}_{\mathrm{f}}$ is the free drug in the supernatant (unentrapped). 


\section{In vitro Drug Release Study}

The in vitro release profile of Apa from BSA-NPs and HA-BSA-NPs was studied using the dialysis method. The NPs were suspended in $1 \mathrm{~mL}$ of PBS and placed in the dialysis bag (VISKING ${ }^{\circledR}$ dialysis tubing MWCO 12,00014,000 ) which was then suspended fully immersed in the release medium (PBS pH 7.4) maintaining sink conditions. The release of Apa was then determined at $37^{\circ} \mathrm{C}$ at $100 \mathrm{rpm}$ in a thermostatically controlled shaking water bath (Köttermann GmbH, Hänigsen, Germany). Samples were collected at predetermined time intervals $(0.25,0.5$, 1, 3, 5, 7 and $24 \mathrm{~h}$ ) then filtered through $0.45 \mu \mathrm{m}$ Millipore $^{\circledR}$ syringe filters, followed by compensation with an equal volume of fresh release medium. The concentration of the drug was determined by the HPLC assay described above. The \% Apa released was calculated in triplicate.

\section{In vitro Mucoadhesion Studies}

The mucoadhesiveness of BSA-NP and HA-BSA-NPs was assessed in vitro by measuring the changes in viscosity and ZP upon formulation incubation with mucin as reported previously. ${ }^{28}$ Mucin was used in a concentration of $10 \%(\mathrm{w} / \mathrm{v})$ with $\mathrm{pH}$ adjusted to 7.4 to mimic the ocular environment. Mucin was first hydrated with distilled water, followed by gentle stirring at room temperature for complete dispersion. Both viscosity and ZP measurements were done in triplicate.

\section{Viscosity Measurements}

BSA-NPs and HA-BSA-NPs were incubated with hydrated mucin in a 1:1 ratio for 30 minutes. NPs control samples were prepared by replacing mucin with distilled water, whereas mucin control sample was prepared similarly but with the NPs being replaced with distilled water. Viscosity of $0.5 \mathrm{~mL}$ samples was measured using cone and plate viscometer (Brookfield, DV2T, USA) following equilibrium for $30 \mathrm{~min}$ at $100 \mathrm{rpm}$ using spindle 40 for 1 min, at $25^{\circ} \mathrm{C}$.

\section{Zeta Potential (ZP)}

NPs interaction with mucin was also determined by measuring the change in $\mathrm{ZP}$ of $\mathrm{NPs} /$ mucin mixtures in the ratio 1:1 compared to NPs and mucin controls. Following incubation for $2 \mathrm{~h}$ at room temperature, ZP was measured as previously described.

\section{In vitro Cell Tolerability Assay on Rabbit Corneal Epithelial Cells (RCE)}

The rabbit corneal epithelial cell line (RCE) was obtained from the European Cell Culture Collection ( $\mathrm{N}^{\circ} 95081046$, ECACC, Salisbury, UK). Cells with passage numbers 10 15 were used. Cells were grown at $37{ }^{\circ} \mathrm{C}$ in a humidified atmosphere with $5 \% \mathrm{CO}_{2}$. The growth medium had the following composition: Dulbecco's modified Eagles medium (DMEM) with Ham's nutrient mixture F12 (1:1), L-glutamine $(1 \% \mathrm{v} / \mathrm{v}, 2 \mathrm{mM})$, penicillin $(100 \mathrm{IU} / \mathrm{mL})$, streptomycin $(0.1 \mathrm{mg} / \mathrm{mL})$, amphotericin B $(0.25 \mu \mathrm{g} / \mathrm{mL})$, epidermal growth factor $(10 \mathrm{ng} / \mathrm{mL})$, insulin $(5 \mu \mathrm{g} / \mathrm{mL})$ and foetal bovine serum, $15 \% \mathrm{v} / \mathrm{v}$. Cytotoxicity test on RCE cells was carried out using a WST-1 commercially available cell proliferation reagent. Cells were plated at $3 \times 10^{5}$ cells/well in 96-well microtiter plates; 24 hours after plating, at $70 \%$ confluence and before the cultures became multilayered, the growth medium was removed and replaced with the test solutions $(100 \mu \mathrm{L})$. After 30 minutes exposure, the reaction medium was removed, the cells were washed twice with DMEM/F12, $100 \mu \mathrm{L}$ fresh growth medium and $10 \mu \mathrm{L}$ cell proliferation reagent WST1 were then added in each well. The cells were incubated for $2 \mathrm{~h}$ at $37{ }^{\circ} \mathrm{C}$ in a humidified atmosphere with $5 \% \mathrm{CO}_{2}$, then the microplate was thoroughly shaken for $1 \mathrm{~min}$ and the absorbance was measured at $450 \mathrm{~nm}$ using a microtiter reader (Asys UVM 340, Biochrom). The time of incubation with the WST-1 reagent was determined after a series of preliminary experiments. The background absorbance was measured on wells containing only the dye solution. The results were expressed as percent optical density of treated vs control untreated, serum-containing cultures.

\section{In vivo Studies}

\section{Animals}

Male Wistar rats, weighing 200-250 g, were provided by the animal facility of the Faculty of Medicine, Alexandria University. Rats were housed at ambient temperature with free access to food and water. Experiments were performed in accordance with the guidelines of the Institutional Animal Care and Use Committee, Faculty of Pharmacy, Alexandria University (Approval number: AU062021299).

\section{Efficacy of BSA-NPs in a Diabetic Retinopathy Rat Model}

For diabetes induction, rats were administered two intraperitoneal doses of streptozotocin (STZ) $(65 \mathrm{mg} / \mathrm{kg}){ }^{29}$ 
Blood glucose levels were measured 3 days after STZ injection and monitored weekly thereafter. Rats were housed for 6 weeks following induction to ensure the development of diabetic retinopathy. Only rats with consistently high glucose levels $>170 \mathrm{mg} / \mathrm{dL}$ were included in the study. Following induction, rats were randomly divided into six groups $(n=5)$ as follows: normal control and positive control groups, two groups treated intravitreally receiving a single $10 \mu \mathrm{L}$ intravitreal injection of ApaBSA-NPs and Apa-HA-BSA-NPs in the right eye following anesthesia by an intraperitoneal injection of ketamine hydrochloride $(20 \mathrm{mg} / \mathrm{kg})$ and two groups treated topically administered $50 \mu \mathrm{L}$ drops of the same formulations twice daily for two weeks. After dose instillation, the eyelids were held open for 10 secs to avoid rapid washout of the formulation. In all animals the left eye was not treated serving as a positive control. Following treatment administration, blood sugar level was measured routinely to make sure it does not exceed $350 \mathrm{mg} / \mathrm{dl}$. At the end of the experiment, the rats were sacrificed, and the eyes were removed immediately for further investigations.

\section{Histological and Morphometric Analysis}

Eyeball specimens were taken for histological and morphometric assessment of the retina. The eyeball was excised and cut into 2 halves. One half was fixed in $10 \%$ formol saline, processed to get $(3-5 \mu \mathrm{m})$ thick paraffin sections and then stained with Haematoxylin and Eosin (H\&E) stain for light microscopic examination. From the other half the retina was excised immediately and cut into small pieces $\left(1 / 2-1 \mathrm{~mm}^{2}\right)$, fixed in $3 \%$ glutaraldehyde solution, and processed to get ultrathin sections for transmission electron microscope (TEM) examination. Digital images from (H\&E) stained sections were obtained at magnification (400X), using a digital camera (Olympus DP20) connected to a microscope (Olympus BX41) for histological examination and measuring the thickness of the retina (RT). TEM (JEOL JEM-2100F; Tokyo, Japan) was used to get images for ultrastructural examination of the retina and to measure the retinal capillary basement membrane (BM) thickness. Measurements were expressed using NIH Image J (v1.49) software as an average of 20 measurements.

\section{Retinal Distribution of BSA-NPs Following Topical and Intravitreal Administration Preparation of Fluorescent Dye Conjugated BSA-NPs Ethanolic solution of $1^{\prime}$-dioctadecyl-3,3,3',3'-}

tetramethylindodicarbocyanine perchlorate (DiD) solution in ethanol $(0.5 \mathrm{mg} / \mathrm{mL})$ was added dropwise to BSA solution in $10 \mathrm{mM} \mathrm{NaCl}$ then the procedure continued as previously mentioned. To remove non-conjugated dye from the NP dispersion, the particles were centrifuged $(15,000$ RPM, 25 min, at $4^{\circ} \mathrm{C}$ ) and re-suspended in PBS.

\section{Retinal Distribution Study}

The study was conducted on both normal and diabetic male Wistar rats divided into groups of three. DiD loaded BSA-NPs and HA-BSA-NPs were administered either topically or intravitreally. Rats in the treated groups were administered a single $10 \mu \mathrm{L}$ dose injected intravitreally in the left eye and a $50 \mu \mathrm{L}$ dose of the same formulation administered topically to the right eye. Five hours post administration, the animals were euthanized by cervical dislocation and their eyes were enucleated and fixed immediately with $10 \%$ formaldehyde solution. Isolated rat retina mounts were then used to visualize the retinal distribution of BSA-NPs by means of Laser scanning confocal microscopy (LSCM) (Leica TSC SPE II/DMi 8) post-administration. Fluorescence was observed with excitation at $545 \mathrm{~nm}$ and emission at 550-600 nm. Confocal images were then analyzed using Image $\mathrm{J}$ software (v 1.49). Mean corrected total fluorescence (MCTF) for identical areas of the analyzed image was calculated by subtracting the background autofluorescence from the measured fluorescence intensity.

\section{Statistical Analysis}

All studies were performed in triplicate. Data were expressed as mean \pm standard deviation (mean $\pm \mathrm{SD}$ ). $t$-test was performed using GraphPad QuickCalcs: $t$-test calculator to determine statistical significance. Differences were considered statistically significant if $p \leq 0.05$.

\section{Results and Discussion Optimization and Physicochemical Characterization of BSA-NPs}

BSA-NPs were prepared by the previously reported desolvation method. ${ }^{25}$ The study of the effect of varying BSA concentration on the physicochemical properties of NPs revealed a significant decrease in particle size (PS) with increasing BSA concentration from 25 to $100 \mathrm{mg}$ which was accompanied by a decrease in PdI at $50 \mathrm{mg}$ followed by a significant increase in PdI at $100 \mathrm{mg}(\mathrm{p} \leq 0.05)$ (Table 1). The observed decrease in PS with increasing 
Table I Composition and Physicochemical Properties of BSA-NPs $(n=3)$

\begin{tabular}{|l|c|c|c|c|c|}
\hline Formula & BSA (mg) & HA (mg) & Particle Size (nm) \pm SD & Pdl & Zeta Potential mV \pm SD \\
\hline BSA-NPI & 25 & - & $260.1 \pm 3.59$ & 0.38 & $-32.3 \pm 0.09$ \\
BSA-NP2 & 50 & - & $212.0 \pm 0.35$ & 0.21 & $-29.5 \pm 0.05$ \\
BSA-NP3 & 100 & - & $100.5 \pm 8.27$ & 0.66 & $-25.7 \pm 0.15$ \\
Apa-BSA-NP & 50 & - & $198.9 \pm 3.52$ & 0.05 & $-29.2 \pm 1.3$ \\
Apa-HA-BSA-NPI & 50 & 1 & $161.3 \pm 6.41$ & 0.39 & $-27.3 \pm 0.05$ \\
Apa-HA-BSA-NP2 & 50 & 2 & $222.2 \pm 3.56$ & 0.22 & $-37.3 \pm 1.8$ \\
Apa-HA-BSA-NP3 & 50 & 4 & $390.0 \pm 2.28$ & 0.19 & $-39.4 \pm 2.0$ \\
\hline
\end{tabular}

BSA concentration is in accordance with the previous investigations of Rahimnejad et al. ${ }^{30}$ who reported that an increase in BSA concentration from 5 to $30 \mathrm{mg} / \mathrm{mL}$ resulted in PS reduction from 204 to $145.7 \mathrm{~nm}$. Oppositely, Galisteo-González and Molina-Bolivar ${ }^{31}$ reported that the increase of BSA concentration from 12 to $100 \mathrm{mg} / \mathrm{mL}$ led to an increase in PS from 75 to $135 \mathrm{~nm}$. The variation in the patterns of change in PS with changing BSA concentration is probably a result of several factors other than BSA concentration working in harmony such as $\mathrm{pH}$, salt concentration, temperature and volume and rate of addition of the dehydrating agent thus affecting protein precipitation by making changes in the environment altering its conformation. ${ }^{32}$

Blank BSA-NPs prepared using $50 \mathrm{mg} / \mathrm{mL}$ and showing an average PS, PdI and ZP of $212 \pm 0.354 \mathrm{~nm}, 0.207$ \pm 0.053 and $-29.5 \pm 0.05 \mathrm{mV}$, respectively, were chosen for further development. Indeed, the optimum protein concentration range for BSA-NPs formulation has been previously reported to be $50-60 \mathrm{mg} / \mathrm{mL} .^{33}$ Apa loading into BSA-NP resulted in a slight reduction in PS (198.9 \pm 3.52 $\mathrm{nm})$ with no change in $\mathrm{ZP}(-29.2 \pm 1.3 \mathrm{mV})$.

To achieve successful coating with $\mathrm{HA}, \mathrm{pH}$ of BSA-NP dispersion was adjusted to 4.9 which represents the isoelectric point (IEP) of BSA at which both the positive and negative charges on the protein surface are equal. At $\mathrm{pH}$ 4.9, ZP shifted from $-29.2 \pm 1.3$ to $6.03 \pm 2.1 \mathrm{mV}$. The negatively charged HA could then be coated onto the surface of the positively charged BSA-NPs through electrostatic interactions. The increase in HA added to BSA-NP dispersion from 1 to $4 \mathrm{mg} / \mathrm{mL}$ (Table 1) resulted in a significant increase in PS with an increase in negative surface charge which prevents particle aggregation as evidenced by the reduction in PdI thus providing long-term stability of the particle suspension ${ }^{19}$ Apa-HA-BSA-NP2 formula coated with $2 \mathrm{mg} / \mathrm{mL} \mathrm{HA}$ and showing PS of
$222.2 \pm 3.56 \mathrm{~nm}$, PdI of 0.22 and $\mathrm{ZP}$ of $-37.3 \pm 1.8 \mathrm{mV}$ was selected for further investigations.

Besides the increase in $\mathrm{ZP}$ observed with increasing HA concentration, the amount of HA that did not bind successfully to the selected HA-coated BSA NPs after centrifugation was determined to ensure efficient HA surface coating by electrostatic interactions onto the surface of Apa-BSA-NP. This was done using the CTAB turbidimetric method. ${ }^{26}$ At the selected initial HA amount (2 mg/ $\mathrm{mL}$ ), free unbound $\mathrm{HA}$ was found to be $2.4 \%$ indicating successful coating of Apa-BSA-NP.

The morphology of Apa-BSA-NP and Apa-HA-BSANP2 was investigated using TEM (Figure 1A). Images displayed solid spheres with smooth surface and narrow distribution (Figure 1A-a and A-c). Apa-HA-BSA-NP2 exhibited a relatively larger size compared to uncoated Apa-BSA-NPs. These results were in agreement with those obtained using zetasizer. The images were further enlarged (Figure 1A-b and A-d) to clearly identify the shape and the morphological structure of the NPs. ApaHA-BSA-NPs appeared as a dark core bounded by a lighter gray halo likely corresponding to the HA coat. Similar observations were reported for HA-based microgels $^{34}$ and HA-coated gold nanoparticles. ${ }^{35}$ The size obtained by TEM was smaller than that obtained by PCS, this has been previously attributed to the sample preparation methods, probably leading to shrinkage of the hydrophilic shell during air-drying and also the contribution of hydrodynamic diameter in PCS measurements. $^{34,36}$

HPLC analysis of unentrapped Apa revealed that the \% EE of Apa calculated for the selected HA-coated BSANPs (Apa-HA-BSA-NP2) was 69 $\pm 1 \%$.

The in vitro drug release profile of Apa from BSA-NPs in PBS pH 7.4 as a function of time is shown in Figure 1B. Both, Apa-BSA-NPs and Apa-HA-BSA-NP2 showed a biphasic release profile with an initial burst 


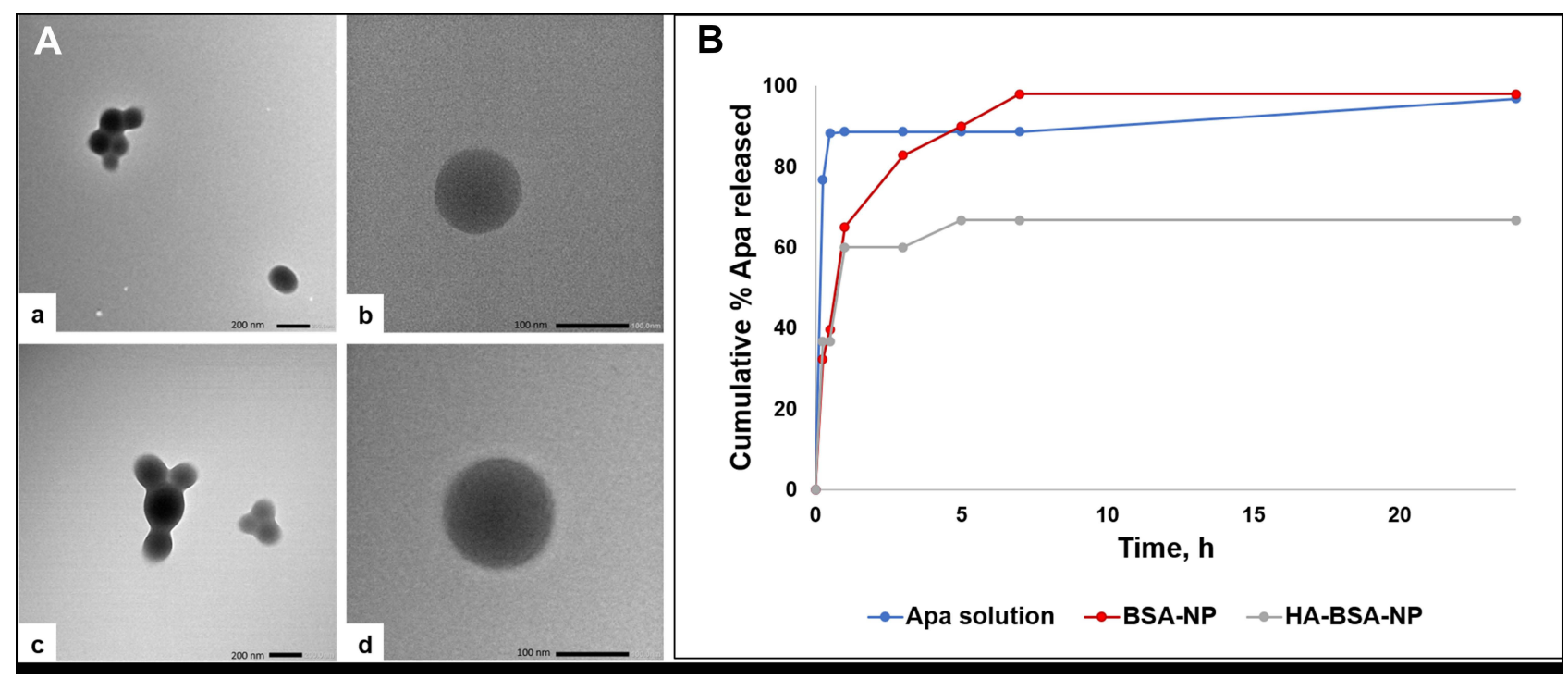

Figure I In vitro characterization of BSA-NPs: (A)TEM images of (a, b) Apa-BSA-NP, (c, d) Apa-HA-BSA-NP at 30K and 50K X magnification, respectively (B) Apatinib release profile from BSA-NP and HA-BSA-NP formulations in PBS pH 7.4 at $37^{\circ} \mathrm{C}$ over $24 \mathrm{~h}(\mathrm{n}=3)$.
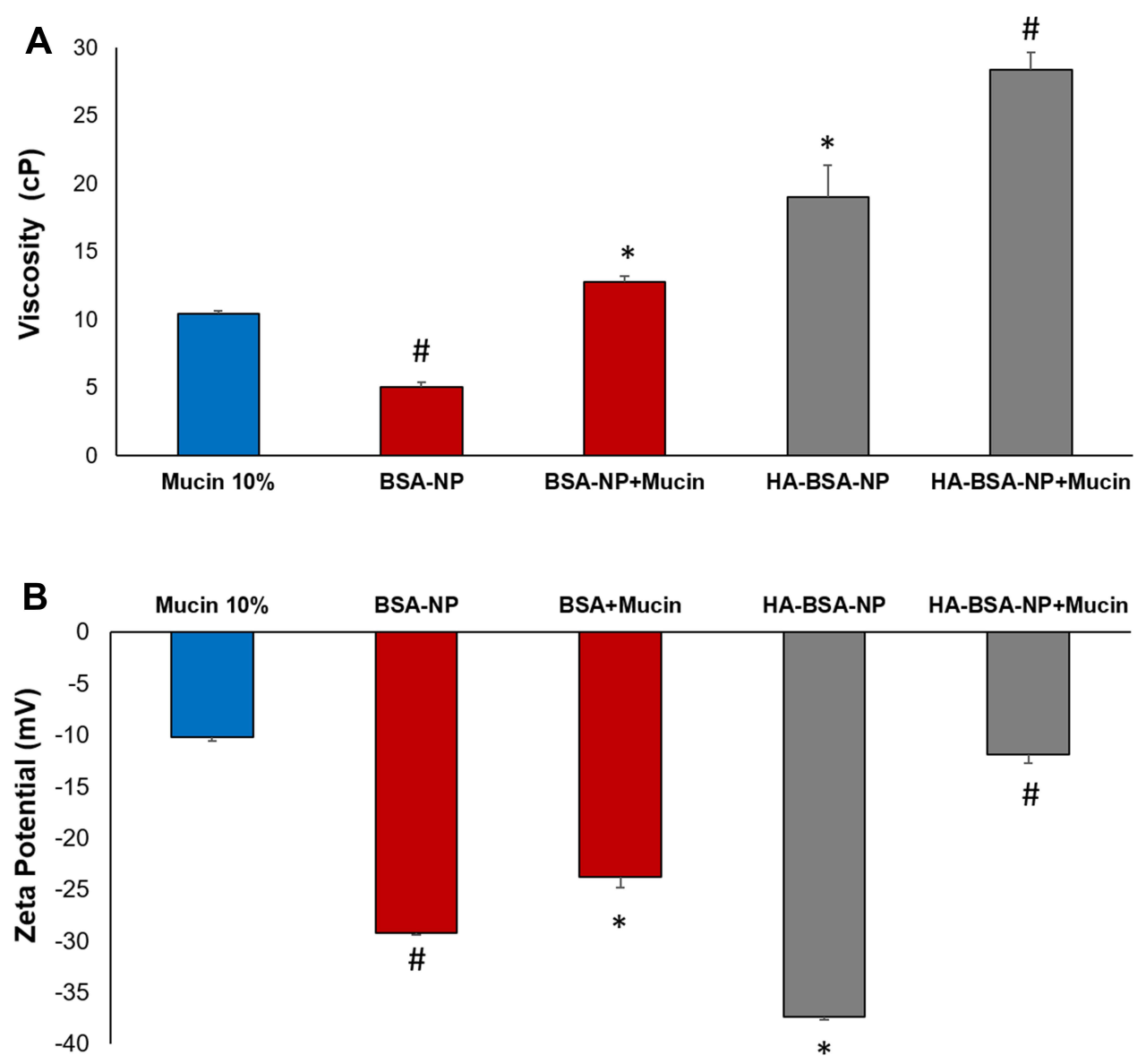

Figure 2 Viscosity $(\mathbf{A})$ and zeta potential $(\mathbf{B})$ values of BSA-NPs and HA-BSA-NPs before and after incubation with mucin as an indicator of mucoadhesion ( $\mathrm{n}=3$ ). ${ }^{*} \mathrm{p}<0.05$ vs BSA-NP, ${ }^{\#}$ < $<0.05$ vs BSA-NP+mucin. 
corresponding to the drug physically bound to the surface followed by sustained release of the entrapped drug. This agrees with the heretofore reported release profiles of aspirin and salicylic acid from albumin NPs showing two distinct phases. ${ }^{14,37}$ Compared to Apa-BSA-NPs, the \% Apa released was reduced significantly by the HA coat (98\% and $66.67 \%$ at $24 \mathrm{~h}$ for Apa-BSA-NPs and Apa-HABSA-NPs, respectively). It has been shown previously that modifying the surface of nanoparticles with HA slows down drug release probably due to restricting release medium diffusion into the NPs matrix. ${ }^{18,38}$ Moreover, HA molecules produce a dense hydrophilic matrix around the nanoparticles, hence decreasing the release rate of hydrophobic drugs. ${ }^{39}$

\section{In vitro Mucoadhesion Study}

As a measurement of mucoadhesive potential, the interaction of NP formulations with mucin was determined by analyzing the changes in viscosity following 30 min incubation. Higher elevation in viscosity values reflects enhanced interaction between the NPs and mucin. ${ }^{28}$ As shown in Figure 2A, HA-BSA-NPs viscosity was significantly higher $(\mathrm{p}<0.05)$ than that of BSA-NPs $(19.01 \pm 2.3$ and $5.05 \pm 0.3 \mathrm{cP}$, respectively). Mucin control viscosity under the selected experimental conditions was $10.5 \pm 0.2 \mathrm{cP}$. Viscosity values of mucin/NPs mixture increased to $12.79 \pm 0.38$ and $28.42 \pm 1.25 \mathrm{cP}$ following incubation with BSA-NPs and HA-BSA-NPs, respectively. Despite the significant increase in viscosity observed for the two tested formulations after mucin addition, the change in viscosity for HA-BSA-NPs was still higher than that observed for the uncoated BSA-NPs (9.41 and $7.74 \mathrm{cP}$, when compared to NPs control, respectively). This could be attributed to the ability of HA linear molecules to penetrate mucin coil structure forming hydrogen bonds with carboxyl and hydroxyl moieties in the amino groups producing a rather stable connection. ${ }^{28,40}$

Changes in $\mathrm{ZP}$ as an indicator of mucin/NPs interaction were also evaluated. As presented in Figure $2 \mathrm{~B}$, mucin particles ZP was about $-10 \mathrm{mV}$. A much higher $\mathrm{ZP}$ was observed for BSA-NP and HA-BSA-NP $(-29.5 \pm 0.05$ and $-37.3 \pm 1.8 \mathrm{mV}$, respectively). Following incubation with mucin the change in ZP was significantly higher with HABSA-NPs than BSA-NPs (25.4 and $5.7 \mathrm{mV}$, respectively compared to NPs controls) to reach $(-11.9 \pm 0.8 \mathrm{mV}$ and $-23.8 \pm 0.98 \mathrm{mV}$, respectively). Similar findings were obtained with the negatively charged HA-coated liquid lipid nanocapsules, ${ }^{41}$ showing a sharp decline in $\mathrm{ZP}$ to a value almost equal to that of mucin. A possible explanation is the formation of a mucin layer covering NPs to a great extent. Even though, HA and mucin carry a negative charge, interaction in this case is not only attributed to electrostatic bonding as despite mucin net negative charge, positively charged regions exist in the non-glycosylated part. Furthermore, the hydrating nature and flexibility of HA enable it to interact with mucin glycosyl groups by the formation of hydrogen bonds. ${ }^{42}$

The above findings confirm the higher degree of interaction of HA-BSA-NP with mucin compared to the uncoated BSA-NP. This consequently results in better adhesion of the topical formulation enhancing ocular contact and increasing pre-corneal residence time when administered topically to the eye.

\section{In vitro Cell Tolerability Assay}

The tolerability of blank and Apa loaded BSA NPs was investigated on RCE cells by WST-1 assay. To test the potential cytotoxicity of BSA NPs, RCE cells were treated with BSA NPs at various concentrations. BSA NPs either blank or Apa loaded did not show any cytotoxicity against RCE cells whose cell viability values were always maintained above $95 \%$; the cells grew well in all the concentrations of BSA NPs tested compared to control. BSA implication in a variety of roles related to the survival and regeneration of cells has been widely investigated. ${ }^{43}$ Huang et al previously reported excellent biocompatibility of BSA NPs on ARPE-19 cells showing no significant difference in cell viability between the free peptide and negative control $(p>0.05)$ after incubation with uncoated and HA-coated FITC-Cx43 MP-HSA NPs. ${ }^{18}$ BSA NPs retinal biocompatibility was also confirmed in vivo in Wistar rats. ${ }^{44}$ The retina, as well as other ocular tissues, kept its cytoarchitecture without showing signs of alteration in the photoreceptor and neuronal layers or cellular infiltrations or vascular inflammation. ${ }^{44}$

\section{In vivo Efficacy of BSA-NPs in Diabetic Retinopathy Rat Model}

Histopathological changes in retinal sections of rats' eyes with DR are shown in Figures 3 and 4. Light microscopic examination of retinal sections from the normal control group showed normal retinal architecture, with its different layers and normal vascularization (Figure $3 \mathrm{~A}-\mathrm{a}$ ). On the other hand, retinal sections from the positive control group (diabetic untreated) showed, increased number of blood 

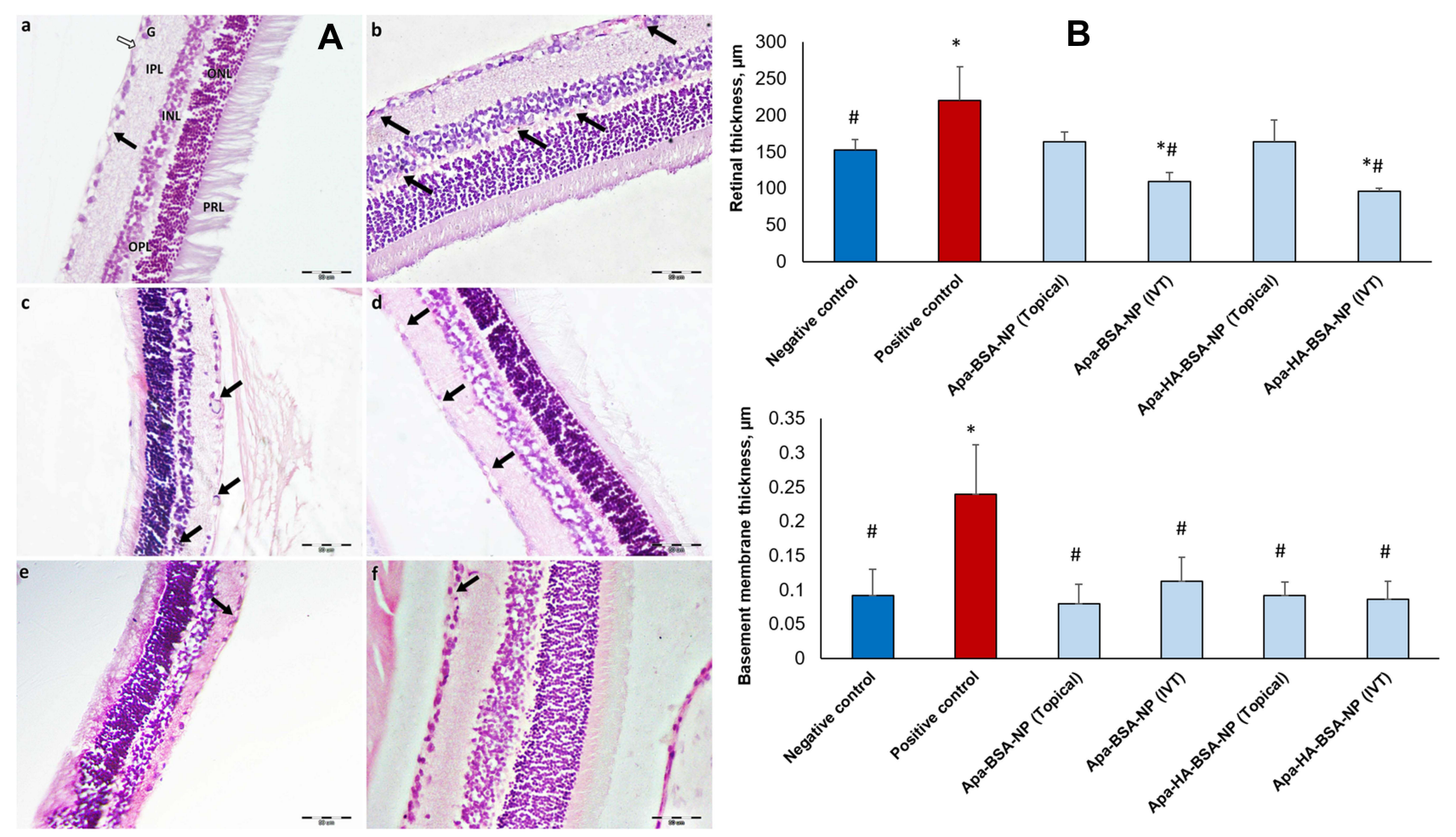

Figure 3 Retinal histology on day 14 following topical and intravitreal administration of Apa-BSA-NPs and Apa-HA-BSA-NPs (A) Histopathological photomicrographs of H\&E stained retinal sections (a) Negative control group showing normal retinal architecture, with its different layers and normal vascularization. (b) Positive control group showing increased number of blood vessels, some of them are dilated and congested. (c) Intravitreal Apa-BSA-NP and (d) Topical Apa-BSA-NPs showing a fewer number of blood vessels than diabetic group. (e) Intravitreal Apa-HA-BSA-NPs and (f) Topical Apa-HA-BSA-NPs showing, almost normal retina. (black arrow); Blood vessels. (white arrow); Nerve fiber layer. (G); Ganglion cell layer. (IPL); Inner plexiform layer. (INL); Inner nuclear layer. (OPL); Outer plexiform layer. (ONL); Outer nuclear layer. (PRL); Photoreceptor layer. (B) Morphometric analysis of full retinal and basement membrane thickness, $\mu \mathrm{m}(\mathrm{n}=20)$. ${ }^{*} \mathrm{p}<0.05$ vs negative control, ${ }^{\#} \mathrm{p}<0.05 \mathrm{vs}$ positive control.

vessels, some of them were dilated and congested (Figure 3A-b). Photomicrographs of retinal sections from groups administered Apa-BSA-NP either topically $(50 \mu \mathrm{L})$ or intravitreally $(10 \mu \mathrm{L})$ showed fewer blood vessels compared to the positive control group (Figure 3A-c and A-d). Moreover, the groups receiving Apa-HA-BSA-NPs showed almost normal retinal vascularization comparable to the normal control group (Figure 3A-e and A-f).

One of the key features of retinal affection in DR is the thickening of the retina. ${ }^{45}$ As shown in Figure 3B, the retinal thickness (RT) of the DR control group (220.24 $\mu \mathrm{m})$ was significantly higher than the normal control group $(151.84 \mu \mathrm{m})$ due to edema and retinovascular dilation. Whereas Apa-BSA-NPs and Apa-HA-BSA-NPs applied either topically or IVT presented a significant decline in RT versus the DR control group, solely topical Apa-HA-BSA-NP formulation showed RT comparable to the control group $(158.97 \mu \mathrm{m}, \mathrm{p}>0.05)$. Moreover, intravitreally injected formulations resulted in a subnormal RT even less than that of the control group. This further decline may be associated with intravitreal injections of anti-VEGF as mentioned by Kniggendorf et al. ${ }^{46}$
TEM imaging of retinal sections (Figure 4) showed findings comparable to those of the light microscopic ones. The electron micrographs of retinal sections from the normal control group showed normal retinal ultrastructure, . The photoreceptor cell layer showed the photoreceptor cells with regular horizontal lamellar discs. Minimal intercellular spaces between nuclei of photoreceptor cells were revealed. Blood vessels showed normal thickness of the basement membrane. On the other hand, those from diabetic group, showed distorted retinal ultrastructure, as the photoreceptor cell layer showed distorted lamellar discs with vacuolations of the outer segments and increased interdisc spaces. In addition, wide spaces were noticed between photoreceptor cells' nuclei. Blood vessels showed thickened basement membrane. These results were in accordance with Gawish et $\mathrm{al}^{47}$ who reported that the retina of diabetic group showed distortion lamellar discs and wide spaces between nuclei of photoreceptor cells. Besides, thickening of the blood vessels is a wellknown finding in diabetic retinopathy. ${ }^{48}$ Intravitreal ApaBSA-NPs group showed areas of distorted lamellar discs and other normal retinal ultra-structure. On the other 


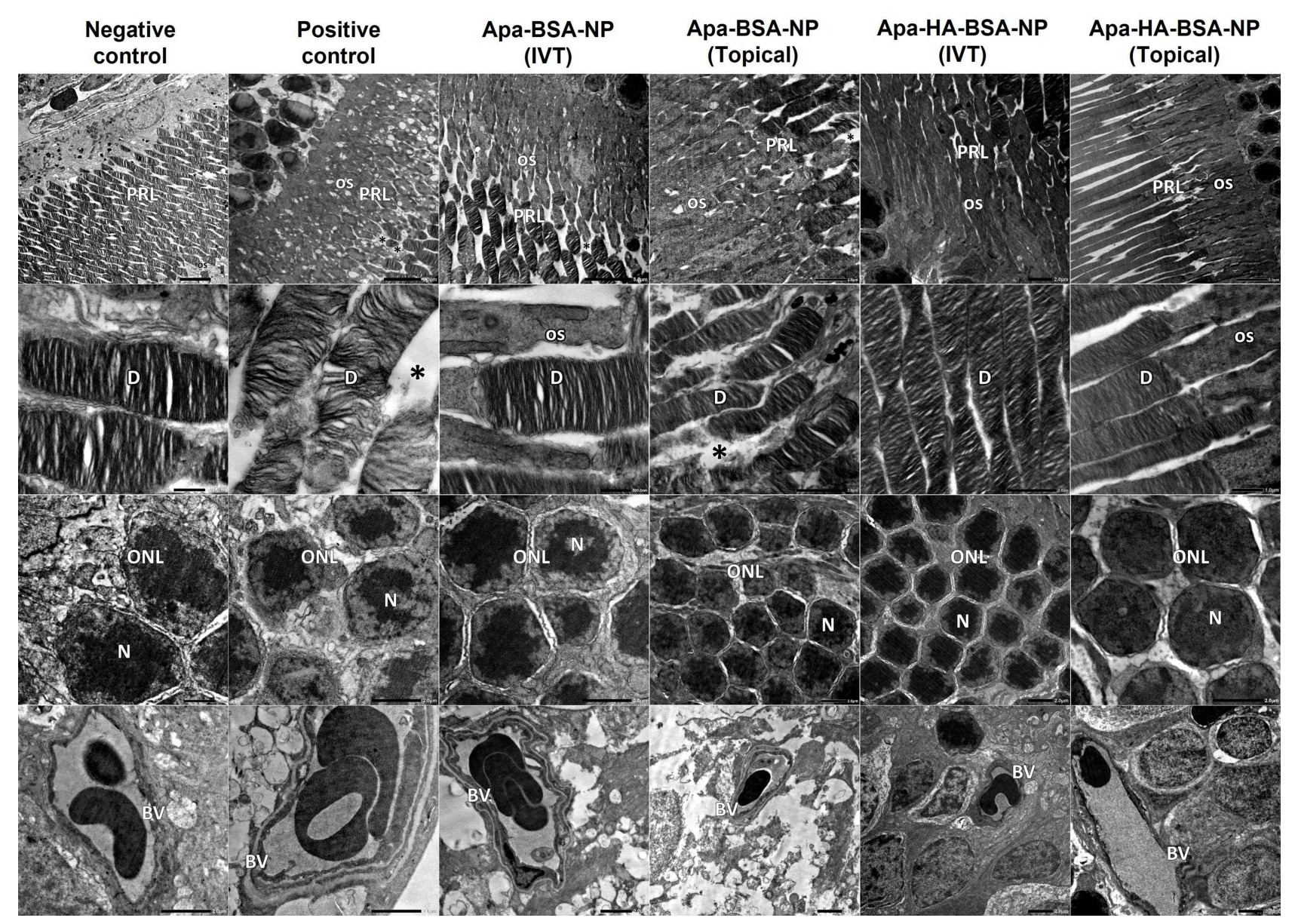

Figure 4 Electron micrographs of retinal sections from negative control show normal organized photoreceptor cell layer (PRL), the photoreceptor cells are with regular horizontal lamellar discs (D). Outer nuclear cell layer (ONL) shows minimal intercellular spaces between nuclei (N) of photoreceptor cells. Normal blood vessel (BV) with normal thickness of the basement membrane is noticed. Electron micrographs from positive control show disorganized photoreceptor cell layer (PRL) with increased interdisc spaces (*), photoreceptor cells show distorted lamellar discs (D) and vacuolated outer segments (OS). ONL shows wide spaces between photoreceptor cells' nuclei (N). Notice BV with thickened basement membrane. Electron micrographs from Apa-BSA-NPs (IVT) group show PRL with few distorted lamellar discs and vacuolated OS and increased interdisc spaces (*), these findings are more prominent in Apa-BSA-NPs (Topical) group. Electron micrographs from Apa-HA-BSA-NPs (IVT) and Apa-HABSA-NPs (Topical) groups show almost normal retinal ultra-structure. All treated groups showed normal BV basement membrane thickness.

hand, the topical Apa-BSA-NPs group showed distorted retinal ultra-structure with only few normal areas. As regard Apa-HA-BSA-NPs groups almost normal retinal ultra-structure was revealed following both intravitreal and topical administration. In all treated groups normal thickness of the basement membrane of the blood vessels was noticed.

The observed changes in the vascular BM thickness were confirmed by morphometrical analysis. Figure 3B illustrates the significant increase in vascular BM thickness in the positive control group compared to the negative control. BM thickening is a fundamental structural alteration of small blood vessels and a histological hallmark of diabetic microangiopathy. Further progression of BM thickening in DR leads to ischemia and retinal capillaries degeneration. ${ }^{49}$ All treatment groups showed a highly significant decrease in BM thickness compared to the DR control group $(p<0.05)$ with the HA-coated NPs, either topical or IVT reaching a comparable thickness to the normal control group $(0.09 \mu \mathrm{m})$.

The results of histopathological examination of rat retinas by both light and electron microscopes demonstrated efficacy of Apa-BSA-NPs in DR rat model following both intravitreal and topical administration. HA coating of BSA-NPs resulted in efficacy enhancement following application by either route. Achieving DR resolution by topical route similar to intravitreal injection and comparable to negative control group is very promising. The mucoadhesive nature of HA as well as its interaction with hyaluronan receptors on corneal epithelial cells may result in prolonged precorneal-retention. ${ }^{50}$ This was previously reported by Kyyrönen et al. where hyaluronic acid 
ester preparations increased the residence time of methylprednisolone in the tear fluid of rabbits. ${ }^{51}$ Likewise, HA coating on chitosan NPs resulted in improved bioavailability of dexamethasone due to its precorneal retentive potential. ${ }^{50}$ Moreover, HA speed up NPs cellular uptake by receptor-mediated endocytosis probably due to its binding to CD44 receptors. ${ }^{52}$ The CD44 receptor is a cell surface glycoprotein and is a receptor for the major extracellular matrix (ECM) component hyaluronan (HA) contributing to its binding, endocytosis, and metabolism. ${ }^{53}$ Increasing evidence has suggested that CD44 play a significant role in the development of various inflammatory diseases and is over expressed in disease conditions. ${ }^{53,54}$ It has been reported that $\mathrm{CD} 44$ receptors affinity for HA enables cells to internalize large HAcoated molecules. This receptor mediated uptake allows negatively charged NPs to still be taken up efficiently by retinal cells. ${ }^{17}$ The improved efficacy of HA-coated BSANPs is thus expected to allow for enhanced Apa delivery to cells overexpressing CD44 receptors enabling receptormediated endocytosis.

In addition to the role of HA coating, NP surface properties have been shown to play a critical role in the diffusive mobility of NPs through the vitreous as well as their penetration across the retina. ${ }^{55}$ The nature of the vitreous humor plays a key role in charge-related particle distribution due to its anionic structure ${ }^{56}$ as it is composed of a three-dimensional network of collagen fibrils bridged by anionic proteoglycans. ${ }^{57}$ This substantially restricts the movement of cationic and large-sized particles after intravitreal injection via hydrophobic, electrostatic, or steric effects while allowing the distribution of anionic particles without any major restrictions. Kim et al. tracked the movement of intravitreally injected human serum albumin nanoparticles as a function of surface charge and retinal injury, showing that anionic nanoparticles readily traversed the vitreous compared to cationic nanoparticles thus reflecting their potential for drug delivery to the subretinal space and the RPE. ${ }^{58}$ The particle characteristics of BSA-NPs prepared in this work, including the highly negative surface charge $(-29.5 \pm 0.05$ and $-37.3 \pm 1.8 \mathrm{mV}$ ) and the relatively small particle size $(212 \pm 0.35$ and $222.2 \pm 3.56 \mathrm{~nm})$ for uncoated and HA-coated NPs, respectively, seemed to avoid particle trapping within the vitreous meshwork allowing them to freely diffuse towards the retina. Huang et al. ${ }^{59}$ investigated HA-coated HSA-NPs biodistribution and retention in vivo showing their rapid diffusion through the vitreous and penetration across the neural retina, till reaching the RPE layer.

\section{Retinal Distribution Study}

Confocal laser microscopy was used to detect the presence of the uncoated and HA-coated BSA-NPs in rat retinal mounts five hours post administration. Fluorescence intensity of different formulations was compared for normal and diabetic rats (Figure 5A). In normal rats no significant difference was observed between the different groups studied $(p>0.05)$ with a mean fluorescence intensity of $2.82 \pm 0.67$

A highly significant increase in fluorescence intensity was observed following IVT administration of both uncoated and HA-coated BSA-NPs in diabetic rats (7.93 \pm 0.93 and $9.42 \pm 1.62$ fold, respectively) compared to normal rats. This could be attributed to remodeling of retinal architecture in DR disrupting ocular physiology. ${ }^{60}$ The retinal blood vessels and the retinal pigment epithelium both act as barriers to fluorescent dye leakage within the retina in a normal eye. Moreover, the tight junctions of the endothelial cells in retinal capillaries and in the retinal pigment epithelium provide an outer blood-retinal barrier preventing penetration into the retinal tissues. ${ }^{60,61}$ On the other hand, in DR fluorescent dye leakage can result from disruption of the retinal vascular endothelial cell tight junctions or the breakdown of tight junctions between retinal pigment epithelial cells. Intense leakage can also be explained by the retinal neovascularization in DR. $^{60,61}$

Regarding topical administration, a significant increase of fluorescence intensity in DR was only observed for HAcoated nanoparticles $(4.3 \pm 0.48$ fold $)$ compared to normal rats (Figure 5B). Moreover, a significantly higher fluorescence intensity in diabetic rats was observed with HAcoated BSA-NPs compared to uncoated NPs $(12.28 \pm 1.39$ and $3.55 \pm 0.81$, respectively, Figure $5 \mathrm{~A}$ ) further elaborating the benefits of HA coating in enhancing ocular drug penetration and diffusion to the posterior segment of the eye via a non-invasive route of administration. A single administration of HA-coated BSA-NPs topically succeeded in achieving a fluorescence intensity of $44 \%$ relative to intravitreal route. This explains the comparative efficacy observed in the in vivo study following repeated daily administration for two weeks. 

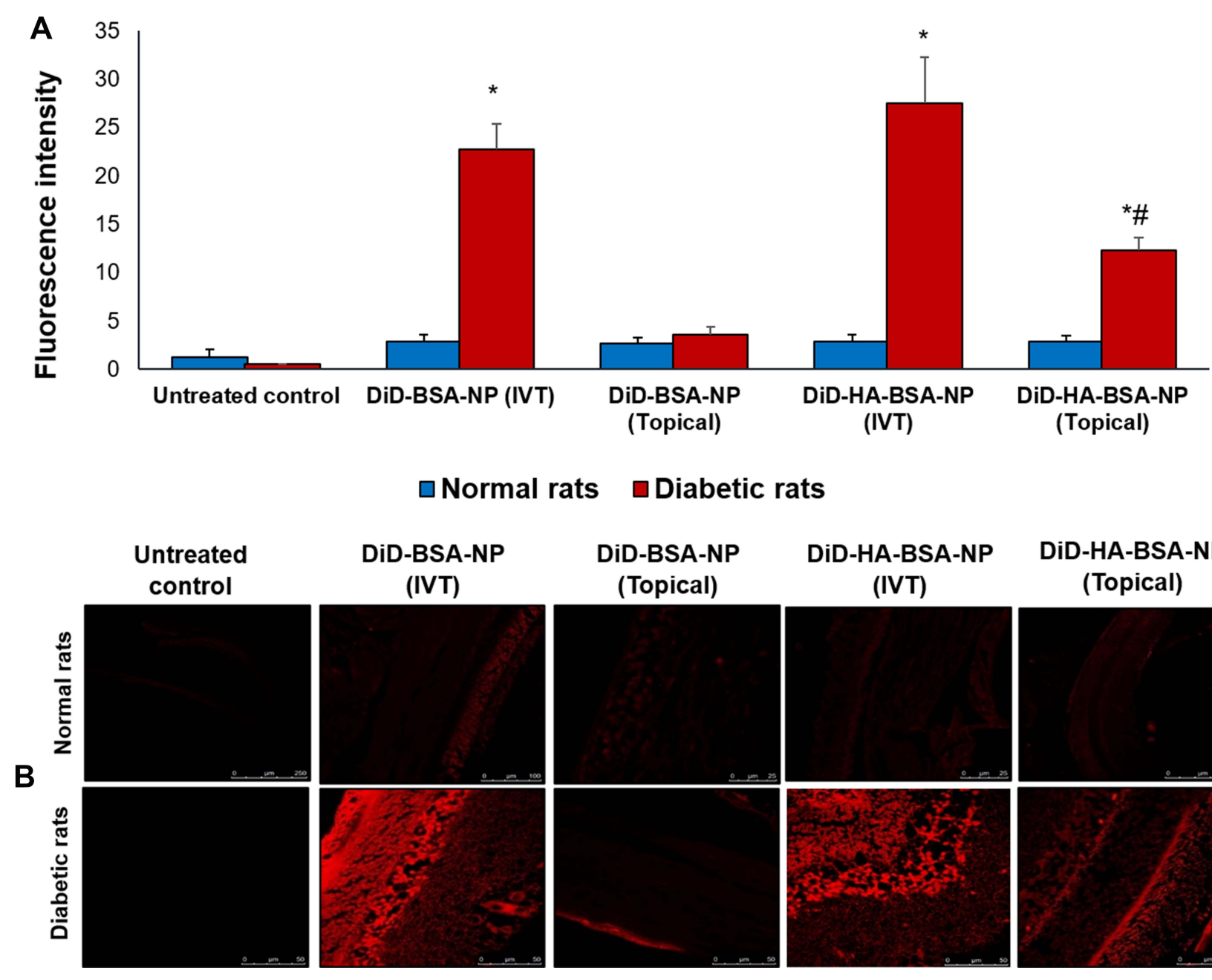
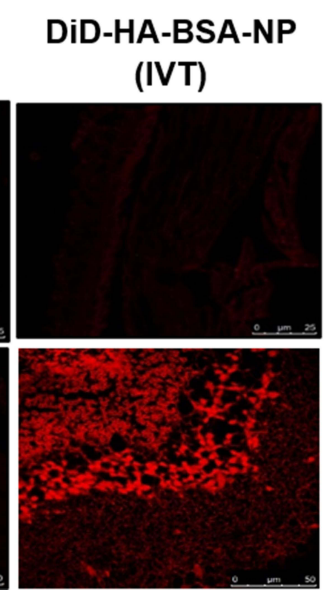

DiD-HA-BSA-NP

(Topical)

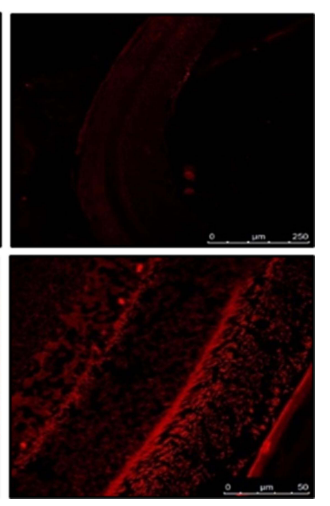

Figure 5 Retinal biodistribution by confocal laser microscopy five hours post single intravitreal and topical application of DiD-BSA-NP and DiD-HA-BSA-NP showing: (A) fluorescence intensity in normal and diabetic rats, (B) fluorescence images, upper panel for normal rats and lower panel for diabetic rats administered (a, b) untreated control, (c, d) intravitreal DiD-BSA-NP, (e, f) topical DiD-BSA-NP, (g, h) intravitreal DiD-HA-BSA-NP and (i, j) topical DiD-HA-BSA-NP. * $<$ <0.05 for diabetic rats vs similarly treated normal rats, ${ }^{\#}<0.05$ for HA-coated NP vs the uncoated NPs administered by the same route.

\section{Conclusion}

Treating DR topically is a challenging objective due to the unique structure of the eye as it combats multiple barriers of various properties rendering the passage of drugs extremely difficult. A delivery system based on HA-coated BSA NPs was developed for the topical administration of Apa - a recently discovered anti VEGF receptor 2. BSA NPs were prepared by the desolvation method and optimized for PS, PdI and ZP. Efficient HA acid coating on selected preformed BSA-NPS based on electrostatic interaction between HA and positively charged BSA-NPs was achieved. In vitro mucoadhesion and in vivo efficacy and biodistribution data obtained implied possible targeting of the posterior eye via the topical route by HA-BSA-NPs. The results obtained promote topical Apa-HA-BSA-NPs as a mucoadhesive, safe alternative to invasive intravitreal route currently adopted for DR treatment with low toxicity as evidenced by the in vitro cytotoxicity study on RCE cell line and put forward a potential therapy with fewer complications and enhanced patient compliance.

\section{Disclosure}

The authors report no conflicts of interest in this work.

\section{References}

1. Bhagat N, Zarbin MA. Epidemiology, risk factors, and pathophysiology of diabetic retinopathy. In: Bandello F, editor. Clinical Strategies in the Management of Diabetic Retinopathy: A Step-By-Step Guide for Ophthalmologists. Cham: Springer International Publishing; 2019:1-19.

2. Lakhani P, Patil A, Majumdar S. Recent advances in topical nano drug-delivery systems for the anterior ocular segment. Ther Deliv. 2018;9(2):137-153. doi:10.4155/tde-2017-0088 
3. Cabrera FJ, Wang DC, Reddy K, Acharya G, Shin CS. Challenges and opportunities for drug delivery to the posterior of the eye. Drug Discov Today. 2019;24(8):1679-1684. doi:10.1016/j.drudis.2019.05.035

4. Suri R, Beg S, Kohli K. Target strategies for drug delivery bypassing ocular barriers. J Drug Deliv Sci Tech. 2020;55.

5. Laradji AM, Kolesnikov AV, Karakocak BB, Kefalov VJ, Ravi N. Redox-responsive hyaluronic acid-based nanogels for the topical delivery of the visual chromophore to retinal photoreceptors. ACS Omega. 2021;6(9):6172-6184. doi:10.1021/acsomega.0c05535

6. Yadav D, Varma LT, Yadav K, et al. Drug delivery to posterior segment of the eye: conventional delivery strategies, their barriers, and restrictions. In: Patel JK, editor. Drug Delivery for the Retina and Posterior Segment Disease. Cham: Springer International Publishing; 2018:51-67.

7. Omerović N, Vranić E. Application of nanoparticles in ocular drug delivery systems. Health Technol (Berl). 2020;10(1):61-78. doi:10.1007/s12553-019-00381-w

8. Karimi M, Bahrami S, Ravari SB, et al. Albumin nanostructures as advanced drug delivery systems. Expert Opin Drug Deliv. 2016;13 (11):1609-1623. doi:10.1080/17425247.2016.1193149

9. Verma D, Gulati N, Kaul S, Mukherjee S, Nagaich U. Protein based nanostructures for drug delivery. $J$ Pharm (Cairo). 2018;2018:9285854. doi:10.1155/2018/9285854

10. Tao C, Chuah YJ, Xu C, Wang D-A. Albumin conjugates and assemblies as versatile bio-functional additives and carriers for biomedical applications. $J$ Mater Chem B. 2019;7(3):357-367. doi:10.1039/C8TB02477D

11. Tan YL, Ho HK. Navigating albumin-based nanoparticles through various drug delivery routes. Drug Discov Today. 2018;23 (5):1108-1114. doi:10.1016/j.drudis.2018.01.051

12. Kim D, Maharjan P, Jin M, et al. Potential albumin-based antioxidant nanoformulations for ocular protection against oxidative stress. Pharmaceutics. 2019;11(7):297. doi:10.3390/pharmaceutics11070297

13. Noomwong P, Ratanasak W, Polnok A, Sarisuta N. Development of acyclovir-loaded bovine serum albumin nanoparticles for ocular drug delivery. Int J Drug Deliv. 2011;3:669-675.

14. Das S, Bellare JR, Banerjee R. Protein based nanoparticles as platforms for aspirin delivery for ophthalmologic applications. Colloids Surf B Biointerfaces. 2012;93:161-168. doi:10.1016/j. colsurfb.2011.12.033

15. Minatelli JA, Stephen Hill W, Moerck RE. Composition and method to alleviate joint pain using low molecular weight hyaluronic acid and astaxanthin. 09795631;15193236. 2017.

16. Naik JB, Pardeshi SR, Patil RP, Patil PB, Mujumdar A. Mucoadhesive micro-/nano carriers in ophthalmic drug delivery: an overview. BioNanoScience. 2020;10(3):564-582. doi:10.1007/ s12668-020-00752-y

17. Martens TF, Remaut K, Deschout H, et al. Coating nanocarriers with hyaluronic acid facilitates intravitreal drug delivery for retinal gene therapy. $J$ Control Release. 2015;202:83-92. doi:10.1016/j. jconrel.2015.01.030

18. Huang D, Chen YS, Rupenthal ID. Hyaluronic acid coated albumin nanoparticles for targeted peptide delivery to the retina. Mol Pharm. 2017;14(2):533-545. doi:10.1021/acs.molpharmaceut.6b01029

19. Huang D, Chen YS, Thakur SS, Rupenthal ID. Ultrasound-mediated nanoparticle delivery across ex vivo bovine retina after intravitrea injection. Eur J Pharm Biopharm. 2017;119:125-136. doi:10.1016/j. ejpb.2017.06.009

20. Huerta Angeles G, Nesporova K. Hyaluronan and its derivatives for ophthalmology: recent advances and future perspectives. Carbohydr Polym. 2021;259:117697. doi:10.1016/j.carbpol.2021.117697

21. Halasz K, Kelly SJ, Iqbal MT, Pathak Y, Sutariya V. Utilization of apatinib-loaded nanoparticles for the treatment of ocular neovascularization. Curr Drug Deliv. 2019;16(2):153-163. doi:10.2174/1567201815666181017095708
22. Lee JE, Kim KL, Kim D, et al. Apatinib-loaded nanoparticles suppress vascular endothelial growth factor-induced angiogenesis and experimental corneal neovascularization. Int $J$ Nanomedicine. 2017;12:4813-4822. doi:10.2147/IJN.S135133

23. Kim KL, Suh W. Apatinib, an inhibitor of vascular endothelial growth factor receptor 2, suppresses pathologic ocular neovascularization in mice. Invest Ophthalmol Vis Sci. 2017;58(9):3592-3599. doi:10.1167/iovs.17-21416

24. Jeong JH, Nguyen HK, Lee JE, Suh W. Therapeutic effect of apatinib-loaded nanoparticles on diabetes-induced retinal vascular leakage. Int J Nanomedicine. 2016;11:3101-3109. doi:10.2147/IJN. S108452

25. Storp B, Engel A, Boeker A, Ploeger M, Langer K. Albumin nanoparticles with predictable size by desolvation procedure. $J \quad$ Microencapsul. 2012;29(2):138-146. doi:10.3109/02652048. 2011.635218

26. Chen Y, Wang Q. Establishment of CTAB turbidimetric method to determine hyaluronic acid content in fermentation broth. Carbohydr Polym. 2009;78(1):178-181. doi:10.1016/j.carbpol.2009.04.037

27. Yu Z, Yu M, Zhang Z, Hong G, Xiong Q. Bovine serum albumin nanoparticles as controlled release carrier for local drug delivery to the inner ear. Nanoscale Res Lett. 2014;9(1):343. doi:10.1186/1556276X-9-343

28. Graca A, Goncalves LM, Raposo S, Ribeiro HM, Marto J. Useful in vitro techniques to evaluate the mucoadhesive properties of hyaluronic acid-based ocular delivery systems. Pharmaceutics. 2018;10 (3):110. doi:10.3390/pharmaceutics 10030110

29. Lai AK, Lo AC. Animal models of diabetic retinopathy: summary and comparison. J Diabetes Res. 2013;2013:106594. doi:10.1155/ 2013/106594

30. Rahimnejad M, Najafpour G, Bakeri G. Investigation and modeling effective parameters influencing the size of BSA protein nanoparticles as colloidal carrier. Colloids Surf a Physicochem Eng Asp. 2012;412:96-100. doi:10.1016/j.colsurfa.2012.07.022

31. Galisteo-Gonzalez F, Molina-Bolivar JA. Systematic study on the preparation of BSA nanoparticles. Colloids Surf B Biointerfaces. 2014;123:286-292. doi:10.1016/j.colsurfb.2014.09.028

32. Tarhini M, Benlyamani I, Hamdani S, et al. Protein-based nanoparticle preparation via nanoprecipitation method. Materials (Basel). 2018;11(3):394. doi:10.3390/ma11030394

33. Jahanban-Esfahlan A, Dastmalchi S, Davaran S. A simple improved desolvation method for the rapid preparation of albumin nanoparticles. Int J Biol Macromol. 2016;91:703-709. doi:10.1016/ j.ijbiomac.2016.05.032

34. Wu Y, Yao J, Zhou J, Dahmani FZ. Enhanced and sustained topical ocular delivery of cyclosporine $\mathrm{A}$ in thermosensitive hyaluronic acid-based in situ forming microgels. Int $J$ Nanomedicine. 2013;8:3587-3601. doi:10.2147/IJN.S47665

35. Apaolaza PS, Busch M, Asin-Prieto E, et al. Hyaluronic acid coating of gold nanoparticles for intraocular drug delivery: evaluation of the surface properties and effect on their distribution. Exp Eye Res. 2020;198:108151. doi:10.1016/j.exer.2020.108151

36. Hu D, Mezghrani O, Zhang L, et al. GE11 peptide modified and reduction-responsive hyaluronic acid-based nanoparticles induced higher efficacy of doxorubicin for breast carcinoma therapy. Int J Nanomedicine. 2016;11:5125-5147. doi:10.2147/IJN.S113469

37. Bronze-Uhle ES, Costa BC, Ximenes VF, Lisboa-Filho PN. Synthetic nanoparticles of bovine serum albumin with entrapped salicylic acid. Nanotechnol Sci Appl. 2017;10:11-21. doi:10.2147/NSA.S117018

38. Hyung W, Ko H, Park J, et al. Novel hyaluronic acid (HA) coated drug carriers (HCDCs) for human breast cancer treatment. Biotechnol Bioeng. 2008;99(2):442-454. doi:10.1002/bit.21578

39. Lee S, Lee CD, Ahn JB, et al. Hyaluronic acid-coated solid lipid nanoparticles to overcome drug-resistance in tumor cells. J Drug Deliv Sci Technol. 2019;50:365-371. doi:10.1016/j.jddst.2019.01.042 
40. Silva B, Marto J, Braz BS, et al. New nanoparticles for topical ocular delivery of erythropoietin. Int $J$ Pharm. 2020;576:119020. doi:10.1016/j.ijpharm.2020.119020

41. Aguilera-Garrido A, Molina-Bolívar JA, Gálvez-Ruiz MJ, GalisteoGonzález F. Mucoadhesive properties of liquid lipid nanocapsules enhanced by hyaluronic acid. J Mol Liq. 2019;296:111965. doi:10.1016/j.molliq.2019.111965

42. Zeng W, Li Q, Wan T, et al. Hyaluronic acid-coated niosomes facilitate tacrolimus ocular delivery: mucoadhesion, precorneal retention, aqueous humor pharmacokinetics, and transcorneal permeability. Colloids Surf B Biointerfaces. 2016;141:28-35. doi:10.1016/j. colsurfb.2016.01.014

43. Horváthy DB, Simon M, Schwarz CM, et al. Serum albumin as a local therapeutic agent in cell therapy and tissue engineering. Biofactors. 2017;43(3):315-330. doi:10.1002/biof.1337

44. Merodio M, Irache JM, Valamanesh F, Mirshahi M. Ocular disposition and tolerance of ganciclovir-loaded albumin nanoparticles after intravitreal injection in rats. Biomaterials. 2002;23(7):1587-1594. doi:10.1016/s0142-9612(01)00284-8

45. Shi Y, Liu Y, Zheng Y, et al. Ethanol extract of chinese propolis attenuates early diabetic retinopathy by protecting the blood-retinal barrier in streptozotocin-induced diabetic rats. J Food Sci. 2019;84 (2):358-369. doi:10.1111/1750-3841.14435

46. Kniggendorf VF, Novais EA, Kniggendorf SL, et al. Effect of intravitreal anti-VEGF on choroidal thickness in patients with diabetic macular edema using spectral domain OCT. Arq Bras Oftalmol. 2016;79(3):155-158. doi:10.5935/0004-2749.20160047

47. Gawish M, Mazen N, Hassen E, Adbelhady M. Light and electron microscopic study on the possible ameliorative role of adipose-derived mesenchymal stem cells on diabetic retinopathy in adult male albino rats. Egypt $J$ Histol. 2018;41(4):582-596. doi:10.21608/ejh.2018.4129.1011

48. Roy S, Maiello M, Lorenzi M. Increased expression of basement membrane collagen in human diabetic retinopathy. J Clin Invest. 1994;93(1):438-442. doi:10.1172/JCI116979

49. Mrugacz M, Bryl A, Zorena K. Retinal vascular endothelial cell dysfunction and neuroretinal degeneration in diabetic patients. J Clin Med. 2021;10(3):458. doi:10.3390/jcm10030458

50. Kalam MA. The potential application of hyaluronic acid coated chitosan nanoparticles in ocular delivery of dexamethasone. Int $J$ Biol Macromol. 2016;89:559-568. doi:10.1016/j. ijbiomac.2016.05.016
51. Kyyrönen K, Hume L, Benedetti L, et al. Methylprednisolone esters of hyaluronic acid in ophthalmic drug delivery: in vitro and in vivo release studies. Int J Pharm. 1992;80(1-3):161-169. doi:10.1016/ 0378-5173(92)90274-6

52. Kalam MA. Development of chitosan nanoparticles coated with hyaluronic acid for topical ocular delivery of dexamethasone. Int $J$ Biol Macromol. 2016;89:127-136. doi:10.1016/j. ijbiomac.2016.04.070

53. Mochimaru H, Takahashi E, Tsukamoto N, et al. Involvement of hyaluronan and its receptor CD44 with choroidal neovascularization. Invest Ophthalmol Vis Sci. 2009;50 (9):4410-4415. doi:10.1167/iovs.08-3044

54. Chandola C, Casteleijn MG, Chandola UM, et al. CD44 aptamer mediated cargo delivery to lysosomes of retinal pigment epithelial cells to prevent age-related macular degeneration. Biochem Biophys Rep. 2019;18:100642. doi:10.1016/j.bbrep.2019.100642

55. Mains J, Wilson CG. The vitreous humor as a barrier to nanoparticle distribution. J Ocul Pharmacol Ther. 2013;29(2):143-150. doi:10.1089/jop.2012.0138

56. Lieleg O, Ribbeck K. Biological hydrogels as selective diffusion barriers. Trends Cell Biol. 2011;21(9):543-551. doi:10.1016/j. tcb.2011.06.002

57. Peng Y, Yu Y, Lin L, et al. Glycosaminoglycans from bovine eye vitreous humour and interaction with collagen type II. Glycoconj J. 2018;35(1):119-128. doi:10.1007/s10719-017-9808-1

58. Kim H, Robinson SB, Csaky KG. Investigating the movement of intravitreal human serum albumin nanoparticles in the vitreous and retina. Pharm Res. 2009;26(2):329-337. doi:10.1007/s11095-0089745-6

59. Huang D, Chen YS, Green CR, Rupenthal ID. Hyaluronic acid coated albumin nanoparticles for targeted peptide delivery in the treatment of retinal ischaemia. Biomaterials. 2018;168:10-23. doi:10.1016/j. biomaterials.2018.03.034

60. Homme RP, Singh M, Majumder A, et al. Remodeling of retinal architecture in diabetic retinopathy: disruption of ocular physiology and visual functions by inflammatory gene products and pyroptosis. Front Physiol. 2018;9:1268. doi:10.3389/fphys.2018.01268

61. Bennett TJ. Available from: https://www.opsweb.org/page/ FAinterpretation?\&hhsearchterms $=\% 22$ angiogram $\% 22$. Accessed June 18, 2021.
International Journal of Nanomedicine

\section{Publish your work in this journal}

The International Journal of Nanomedicine is an international, peerreviewed journal focusing on the application of nanotechnology in diagnostics, therapeutics, and drug delivery systems throughout the biomedical field. This journal is indexed on PubMed Central, MedLine, CAS, SciSearch ${ }^{\circledR}$, Current Contents ${ }^{\mathbb{R}} /$ Clinical Medicine, $^{-}$
Journal Citation Reports/Science Edition, EMBase, Scopus and the Elsevier Bibliographic databases. The manuscript management system is completely online and includes a very quick and fair peer-review system, which is all easy to use. Visit http://www.dovepress.com/ testimonials.php to read real quotes from published authors. 